\section{BIOCONSERVACIÓN FRENTE A PATÓGENOS DE TRANSMISIÓN ALIMENTARIA EN FRUTAS $Y$ HORTALIZAS MÍNIMAMENTE PROCESADAS}

\author{
Isabel Alegre Vilas \\ Universitat de Lleida \\ ORCID iD: https://orcid.org/0000-0002-8153-624X \\ isabel.alegre@udl.cat \\ Maribel Abadias Seró \\ Institute of Agrifood Research and Technology (IRTA) \\ ORCID iD: https://orcid.org/0000-0003-0113-8979 \\ isabel.abadias@irta.cat \\ Pilar Colás Medà \\ Universitat de Lleida \\ ORCID iD: https://orcid.org/0000-0003-3908-7900 \\ pilar.colas@udl.cat \\ Cyrelys Collazo Cordero \\ Universitat de Lleida \\ ORCID iD: https://orcid.org/0000-0002-2633-2332 \\ ccollazo@tecal.udl.cat \\ Inmaculada Viñas Almenar \\ Universitat de Lleida \\ ORCID iD: https://orcid.org/0000-0001-5182-2520 \\ inmaculada.vinas@udl.cat
}

Cómo citar este artículo/Citation: Alegre Vilas, I., Abadias Seró, M. , Colás Medà, P., Collazo Cordero, C. y Viñas Almenar, I. (2020). Bioconservación frente a patógenos de transmisión alimentaria en frutas y hortalizas mínimamente procesadas. Arbor, 196 (795): a543. https://doi.org/10.3989/arbor.2020.795n1007

Recibido: 26 febrero 2019. Aceptado: 29 octubre 2019.

RESUMEN: El aumento en la producción y consumo de frutas y hortalizas mínimamente procesadas de los últimos años ha contribuido a incrementar las toxiinfecciones alimentarias asociadas al consumo de productos vegetales frescos. Esto es debido a que los tratamientos desinfectantes llevados a cabo actualmente por industria de IV gama son insuficientes para garantizar la seguridad microbiológica de los productos finales, y además estos no reciben ningún tratamiento capaz de eliminar todos los patógenos antes de su consumo. Por lo tanto, es necesario utilizar estrategias alternativas para reducir los microorganismos patógenos y alterantes en frutas y hortalizas. La bioconservación, mediante el uso de microorganismos o sus metabolitos, es una alternativa que, en combinación con las prácticas habituales, puede reducir o prevenir el crecimiento de patógenos en productos mínimamente procesados, mejorando su calidad microbiológica. En este artículo se presentan los resultados más relevantes sobre la bioconservación de frutas y hortalizas mínimamente procesadas.

PALABRAS CLAVE: cultivos protectores; bacteriocinas; bacteriófagos; IV gama; seguridad microbiológica.

\section{BIOPRESERVATION AGAINST FOODBORNE PATHOGENS ON MINIMALLY PROCESSED FRUITS AND VEGETABLES}

Copyright: (C) 2020 CSIC. Este es un artículo de acceso abierto distribuido bajo los términos de la licencia de uso y distribución Creative Commons Reconocimiento 4.0 Internacional (CC BY 4.0).

ABSTRACT: The production and consumption of minimally processed fruits and vegetables have increased in recent years, which has contributed to increasing the foodborne illnesses associated with the consumption of fresh produce. This is because current disinfection methods used in the fresh-cut industry are not enough to guarantee the microbial safety of the final products, and minimally processed produce does not receive any other treatment capable of eliminating all pathogens before consumption. Therefore, alternative strategies to reduce pathogenic and spoilage microorganisms are needed. Biopreservation, using microorganisms or their metabolites, is an alternative that, in combination with current practices, could reduce or prevent pathogenic growth in minimally processed produce, improving their microbiological quality. This paper reviews the most relevant results on biopreservation of minimally processed fruits and vegetables.

KEYWORDS: protective cultures; bacteriocin; bacteriophage; fresh-cut; microbial safety. 


\section{INTRODUCCIÓN}

El consumo de frutas y hortalizas es beneficioso para la salud por su contenido en vitaminas, minerales, fibras y sustancias fitoquímicas, que contribuyen a la salud. Asimismo, su consumo puede llegar a reducir el riesgo de mortalidad por enfermedades cardiovasculares y cáncer (Wang et al., 2014).

Las frutas y hortalizas mínimamente procesadas surgen como respuesta a la demanda del consumidor de productos frescos, sanos, de elevada calidad y que requieran escaso tiempo de preparación conservando sus propiedades originales desde el punto de vista organoléptico y nutritivo. Según valoraciones del año 2017 (véase "La IV gama de frutas y hortalizas espera crecer un 10 \% hasta 2020"), en España, el consumo de este tipo de productos ronda los $3 \mathrm{~kg}$ per cápita/ año. Aunque la demanda no es muy alta, en nuestro país más del $70 \%$ de los hogares ha incorporado en alguna ocasión estos productos a la cesta de la compra. De hecho, la industria de IV gama ha crecido continuamente desde su introducción en el mercado español en 1989 y se estima que, para el año 2020, la venta al por menor de productos mínimamente procesados crecerá entre un 8 y un $10 \%$, alcanzando los 320.000 millones de euros en ventas (véase "La IV gama de frutas y hortalizas espera crecer un $10 \%$ hasta 2020").

Las frutas y hortalizas mínimamente procesadas son frutas y hortalizas frescas, limpias, troceadas y envasadas, listas para su consumo, con una fecha de caducidad en torno a los 5-7 días conservadas entre $1-4{ }^{\circ} \mathrm{C}$, que garantizan su seguridad y calidad nutricional y sensorial. El procesado de elaboración de productos de IV gama incluye operaciones como el pelado y el corte, que producen estrés físico en el vegetal y darán lugar a la liberación de fluidos celulares, causando alteraciones fisiológicas y microbiológicas que pueden repercutir en la calidad y vida útil del producto obtenido. En este sentido, uno de los principales retos para la industria de IV gama es la búsqueda de estrategias que permitan conservar la calidad de las frutas y hortalizas cortadas, alargar su vida útil y, sobre todo, garantizar su seguridad.

La microbiota natural de frutas y hortalizas frescas no suele ser patógena para los humanos. Sin embargo, durante el cultivo, la cosecha, el transporte y el posterior procesado y manejo, el producto puede contaminarse con patógenos de transmisión alimentaria, ya sean de origen humano, animal o ambiental (Sánchez, Elizaquível y Aznar, 2012). Esta contaminación con patógenos de transmisión alimentaria plan- tea un posible problema de seguridad alimentaria, ya que los productos mínimamente procesados se consumen crudos, sin ningún tipo de tratamiento microbiológicamente letal. Por todo ello, para evitar y minimizar los riesgos de contaminación de las frutas y hortalizas, es necesario llevar a cabo unas Buenas Prácticas de Producción y de Fabricación y aplicar un sistema de Análisis de Peligros y Puntos de Control Crítico (APPCC).

En el año 2017 se detectaron 39 brotes de toxiinfección alimentaria asociados al consumo de alimentos de origen no animal en la Unión Europea, lo que representa el 6,1\% de los brotes, siendo Salmonella spp. y los Calicivirus, incluyendo los norovirus los principales agentes causales, seguidos de las toxinas bacterianas y de Escherichia coli, productor de Shigatoxina (EFSA and ECDC, 2018). Asimismo, el número de brotes de toxiinfecciones alimentarias causadas por la ingesta de productos mínimamente procesados se sitúa en una tasa bastante alta tanto en Estados Unidos como en la Unión Europea.

Por lo tanto, los métodos de producción seguros y los procedimientos adecuados de descontaminación son pasos críticos para garantizar la seguridad de las frutas y hortalizas frescas listas para el consumo (Artés, Gómez, Aguayo, Escalona y Artés-Hernández, 2009). El lavado de los productos vegetales con agua elimina suciedad, exudados y células muertas que contribuyen al deterioro de la fruta. Sin embargo, para eliminar o reducir la microbiota presente se suelen emplear desinfectantes químicos, con los que además se evita la contaminación cruzada entre productos limpios y contaminados. De hecho, su efecto principal es mantener la calidad microbiana del agua (Gil, Selma, López-Gálvez y Allende, 2009). Aunque el uso de desinfectantes químicos es habitual en la industria de IV gama, los consumidores tienen una percepción negativa de los mismos, ya que algunos de ellos están relacionados con la producción de compuestos tóxicos, y además precisan períodos de tiempo bastante largos para degradarse, creando problemas de contaminación ambiental (Faleiro, 2010). Por ese motivo se buscan alternativas para el control de microorganismos e incrementar la seguridad alimentaria.

En las alternativas para mejorar la seguridad de estos productos, encontramos una gran variedad de métodos físicos, como por ejemplo la luz ultravioleta (UV-C), la alta presión hidrostática, los ultrasonidos y la irradiación, entre otros (Ramos, Miller, Brandao, Teixeria y Silva, 2013). Sin embargo, la mayoría de estas técnicas todavía no están implantadas en la in- 
dustria, siendo el envasado en atmósfera modificada y la refrigeración los métodos físicos más ampliamente aplicados, ya que su combinación evita el desarrollo de microorganismos y retarda la senescencia del producto, alargando la vida útil de las frutas y hortalizas de IV gama.

Otra de las alternativas es la bioconservación, ya sea mediante el uso de microorganismos o sus metabolitos. Estos cultivos protectores ejercen su función ocupando el mismo nicho que los patógenos (compitiendo por espacio y nutrientes), produciendo sustancias antimicrobianas, y en caso de algunas bacterias, como las probióticas, actuando a nivel del intestino impidiendo que los patógenos se adhieran e invadan los enterocitos y activando una respuesta inmunitaria como barrera defensiva contra los patógenos (Fliss, Hammami y Le Lay, 2011). Sin embargo, la bioconservación no sustituye el uso de buenas prácticas de higiene, de forma que siempre se debe considerar como un parámetro adicional para mejorar la seguridad y la calidad del producto (Holzapfel, Geisen y Schillinger,1995).

\section{CULTIVOS PROTECTORES O AGENTES DE BIOCONTROL}

Los cultivos protectores pueden evitar el crecimiento de patógenos de transmisión alimentaria en frutas y hortalizas mínimamente procesadas o pueden incluso ser una alternativa a los tratamientos químicos habituales en la industria de IV gama. Pueden realizarse mediante la utilización de microorganismos previamente existentes en el alimento o mediante la introducción artificial de microorganismos con capacidad de controlar a los patógenos (Leverentz et al., 2003).

\subsection{Microorganismos epifitos}

La microbiota natural de frutas y hortalizas juega un papel importante en la seguridad microbiológica de los productos mínimamente procesados, ya que puede competir con los patógenos por espacio físico y nutrientes o produciendo compuestos antimicrobianos (Liao y Fett, 2001). La desinfección que tiene lugar durante el procesado de estos productos reduce esta microbiota protectora, por lo que, si ocurriera una contaminación posterior con patógenos, estos se encontrarían en una situación ventajosa. Por ejemplo Francis y O'Beirne (1997) observaron que la desinfección de lechuga mínimamente procesada favoreció el crecimiento de Listeria monocytogenes a lo largo de la conservación. Una manera de reducir esta ventaja competitiva es la aplicación de cepas seleccionadas de microorganismos presentes de forma natural en los productos. Siguiendo esta estrategia, Weiss, Hertel, Grothe, Ha y Hammes (2007) observaron que Pseudomonas putida LTH 5878 redujo la viabilidad de Salmonella Typhimurium, Listeria innocua y Staphylococcus aureus en lechuga tras ocho días de conservación a $4{ }^{\circ} \mathrm{C}$. Sin embargo, la presencia de Pseudomonas fluorescens, Lactobacillus plantarum y Pantoea agglomerans en hojas de col no afectó a la población de Listeria monocytogenes durante la conservación a 4 y $30{ }^{\circ} \mathrm{C}$ (Ongeng, Ryckeboer, Vermeulen y Devlieghere, 2007). Posteriormente, Oliveira, Abadias, Colás-Medà, Usall y Viñas (2015) aislaron una cepa de Pseudomonas spp. (M309) que redujo la población de Salmonella Michigan, Escherichia coli 0157:H7 y Listeria monocytogenes, en discos de lechuga durante nueve días de conservación a $10{ }^{\circ} \mathrm{C}$, pero no fue efectiva en condiciones semi-comerciales.

En frutas mínimamente procesadas, Leverentz et al. (2006), observaron que cuatro cepas aisladas de la superficie de la fruta redujeron la población de Listeria monocytogenes en cilindros de manzana conservados a 10 y $25^{\circ} \mathrm{C}$, de las cuales tres también redujeron la de Salmonella Poona a $25^{\circ} \mathrm{C}$ y ninguna a $10^{\circ} \mathrm{C}$. Posteriormente, Abadias, Usall, Alegre, Torres y Viñas (2009) observaron que Candida sake CPA-1 no redujo la población de Escherichia coli en cilindros de manzana. Sin embargo, la cepa CPA-6, nueva especie dentro del género Enterobacteriaceae, y la cepa CPA-7, identificada como Pseudomonas graminis, mostraron actividad antagonista frente a Escherichia coli 0157:H7, Salmonella Michigan y Listeria innocua en cilindros de manzana y melocotón conservados a $20^{\circ} \mathrm{C}$ y frente a Escherichia coli 0157: $\mathrm{H7}$ a $5{ }^{\circ} \mathrm{C}$ (Alegre, Viñas, Usall, Anguera, Figge y Abadias, 2012; Alegre, Viñas, Usall, Teixidó, et al. 2013). Asimismo, el trabajo realizado con CPA-7 sugirió que su modo de acción es competición, un modo de acción al que difícilmente los patógenos pueden desarrollar resistencia. Por todo ello, el antagonista ha sido patentado a nivel europeo (EP-2886665) (Viñas et al., 2017) y americano (US-8735136) (Viñas et al., 2014). Posteriormente, Alegre, Viñas, Usall, Anguera et al. (2013) comprobaron que Pseudomonas graminis CPA-7 era efectivo frente a Salmonella y Listeria monocytogenes en manzana mínimamente procesada en condiciones semi-comerciales conservada a 5 y $10{ }^{\circ} \mathrm{C}$, es decir en presencia de un antioxidante comercial, NatureSeal ${ }^{\circledR}$ AS1, y envasado en atmósfera modificada pasiva. Además, la aplicación del agente de biocontrol no afectó a la calidad del producto. Sin embargo, el efecto observado no fue instantáneo y, por 
lo tanto, debería usarse en combinación con otras técnicas de conservación, como parte de la tecnología de barreras. En melón piel de sapo conservado en condiciones semi-comerciales, la actividad antagonista de Pseudomonas graminis CPA-7 disminuyó con el envasado en atmósfera modificada pasiva (Abadias et al., 2014), ya que es aerobio estricto y su efectividad se reduce en ausencia de oxígeno. En melón galia almacenado durante cinco días a $5{ }^{\circ} \mathrm{C}$ en condiciones aerobias, CPA-7 redujo hasta 1,9 unidades logarítmicas la población de Listeria monocytogenes, mientras que no se observó efecto en melón cantaloupe en las mismas condiciones. Las diferencias en la efectividad se atribuyeron al efecto que la distinta composición de las frutas puede tener en la competencia por nutrientes (Collazo, Abadias, Aguiló-Aguayo et al., 2017). El estudio de la capacidad de CPA-7, Escherichia coli y SalmoneIla spp. de emplear fuentes comunes de carbono presentes en las frutas, sugirió que una mayor eficacia metabólica del antagonista en función de la disponibilidad de nutrientes pudiera brindarle una ventaja adaptativa frente a los patógenos. Iglesias, López, Echeverría, Viñas, Zudaire y Abadias, (2018) demostraron que Pseudomonas graminis CPA-7 fue efectivo frente Salmonella y Listeria monocytogenes en pera conservada a 5,10 y $20{ }^{\circ} \mathrm{C}$, mientras que no fue efectivo en condiciones semi-comerciales. Tras optimizar el tratamiento antioxidante y el film de envasado para simular las condiciones semi-comerciales (Iglesias, Abadias, Anguera y Viñas, 2018), Pseudomonas graminis CPA-7 redujo la población de Listeria monocytogenes pero no la de Salmonella, ya que la población de este patógeno se redujo a lo largo de la conservación por la baja temperatura.

Los estudios in vitro sobre modo de acción de CPA-7 mostraron que no produjo alginato ni formó biopelículas sobre superficies inertes en las condiciones estudiadas. Tampoco se observó actividad antimicrobiana del crecimiento de CPA-7 o de los metabolitos producidos por este en medio sólido, contra Escherichia coli, Salmonella spp. o Listeria monocytogenes (Collazo, Abadias, Aguiló-Aguayo et al., 2017). Por otro lado, CPA-7 demostró tener un efecto sobre la virulencia de Salmonella Enteritidis y Listeria monocytogenes tras la co-incubación en pera mínimamente procesada durante siete días a $5{ }^{\circ} \mathrm{C}$, ya que redujo la adherencia de Salmonella a células Caco-2 (modelo celular del epitelio intestinal humano), mientras que no mostró efecto en la de Listeria monocytogenes. Sin embargo, la interacción con el antagonista en la matriz alimentaria durante el almacenamiento redujo significativamente la invasividad de ambos patógenos: en 1,3 unidades logarítmicas en el caso de Listeria monocytogenes y a niveles indetectables ( $<5$ ufc g $^{-1}$ fruto) en el caso de Salmonella Enteritidis, sugiriendo la interferencia con la expresión de genes involucrados en los mecanismos de virulencia de estos patógenos como posible mecanismo de acción de CPA-7 (Collazo, Abadias, Colás-Medà et al., 2017). Otro posible modo de acción de CPA-7 puede ser la activación de la respuesta defensiva de la planta y la tolerancia al estrés oxidativo, ya que esta bacteria indujo la actividad de enzimas antioxidantes y retardó la reducción del contenido en compuestos antioxidantes en manzana mínimamente procesada a lo largo de seis días de almacenamiento a $5{ }^{\circ} \mathrm{C}$, sin afectar negativamente a la calidad del fruto (Collazo et al., 2018).

\subsection{Bacterias ácido-lácticas}

Las bacterias ácido-lácticas pueden utilizarse como cultivos protectores en alimentos como las frutas y hortalizas mínimamente procesadas. Por ejemplo, Vescovo, Torriani, Orsi, Macchiarolo y Scolari (1996)demostraron el potencial de una bacteria láctica, una cepa de Lactobacillus casei, para inhibir el crecimiento de bacterias en vegetales mínimamente procesados. Posteriormente, Torriani, Scolari, Dellaglio y Vescovo (1999) observaron que la cepa IMPC LP4 de Lactobacillus plantarum aumentó la vida útil de las zanahorias ralladas, controlando el crecimiento de Leuconostoc spp. Scolari y Vescovo (2004) observaron un efecto inhibitorio importante de Lactobacillus casei LC34GF frente a Staphylococcus aureus, Aeromonas hydrophila, Escherichia coli y Listeria monocytogenes en hojas de endivia conservadas durante seis días a $8{ }^{\circ} \mathrm{C}$. Trias, Bañeras, Badosa y Montesinos (2008) seleccionaron cinco bacterias ácido-lácticas con actividad bacteriostática frente a Salmonella y bacteriocida frente a Listeria monocytogenes en lechuga, mientras que solo una de ellas fue bacteriostática frente a Escherichia coli. Los autores atribuyeron la especificidad de las bacterias lácticas por la bacteria Gram positiva ensayada a la producción de bacteriocinas. Siroli et al. (2015) observaron que Lactobacillus plantarum V7B3 aumentó la vida útil y seguridad en manzana y lechuga, incrementando la cinética de destrucción de Escherichia coli y reduciendo la viabilidad de Listeria monocytogenes.

Una opción interesante respecto a la aplicación de bacterias ácido-lácticas es la utilización de bac- 
terias probióticas, ya que además de conseguir un efecto protector en los alimentos podrían mejorar la salud del consumidor. Además, el desarrollo de alimentos probióticos no lácteos podría ampliar el rango de población que se beneficiaria de su consumo. En esta línea, el probiótico Lactobacillus rhamnosus GG mantuvo la concentración necesaria para ejercer efectos beneficiosos en trozos de manzana mínimamente procesados en condiciones semi-comerciales a 5 y $10{ }^{\circ} \mathrm{C}$, y además redujo la población de Listeria monocytogenes en una unidad logarítmica sin afectar a la calidad final del producto (Alegre, Viñas, Usall, Anguera y Abadias, 2011). Russo et al. (2014) y Russo et al. (2015) aplicaron dos cepas potencialmente probióticas (Lactobacillus plantarum B2 CECT 8328 y Lactobacillus fermentum PBCC11.5 CECT 8448) en piña y melón cantaloupe mínimamente procesados. Ambos microorganismos mantuvieron su concentración por encima del nivel mínimo para ejercer su actividad beneficiosa y redujeron la población de Listeria monocytogenes en ambas frutas. Posteriormente, Iglesias, Abadias, Anguera, Sabata y Viñas (2017) observaron que Lactobacillus rhamnosus GG redujo la población de Salmonella y Listeria monocytogenes en pera tras la conservación a 20, 10 y $5{ }^{\circ} \mathrm{C}$ en condiciones semi-comerciales. Asimismo, Lactobacillus rhamnosus GG redujo la adhesión e invasión de Listeria monocytogenes CECT 4032 al epitelio intestinal humano empleando el modelo celular Caco-2 (Iglesias, Viñas, Colás-Medá, Collazo, Serrano y Abadias, 2017) tras su co-inoculación en pera y su conservación a $10{ }^{\circ} \mathrm{C}$ durante siete días. LactobaciIlus rhamnosus no afectó a la calidad de la pera (Iglesias, Echeverría, Viñas, López y Abadias, 2018).

Por lo tanto, el uso de cultivos protectores puede representar un obstáculo adicional al crecimiento de patógenos de transmisión alimentaria en frutas y hortalizas mínimamente procesadas. Asimismo, los trabajos realizados con algunos cultivos protectores han demostrado una reducción en la patogenicidad de los patógenos ensayados.

\section{BACTERIOCINAS}

Como ya se ha comentado, uno de los mecanismos de acción por los cuales los cultivos bioconservantes pueden controlar microorganismos alterantes o patógenos en los alimentos es la producción de metabolitos como las bacteriocinas. Las bacteriocinas son péptidos con actividad antimicrobiana producidos por un gran número de bacterias (Cotter, Hill y Ross, 2005). Principalmente actúan sobre la membrana de las cé- lulas sensibles, desestabilizando y permeabilizando mediante la formación de canales. Se clasifican según sus características bioquímicas y genéticas, diferenciando bacteriocinas de tipo I, II, III y IV (Mondragón Preciado et al., 2013). Es importante destacar que las bacteriocinas son compuestos seguros para los humanos debido a que las proteasas presentes en el tracto gastrointestinal humano las descomponen en sustancias no tóxicas (Chen y Hoover, 2003).

La aplicación de las bacteriocinas como método de bioconservación se ha estudiado ampliamente en productos de origen animal; sin embargo, su aplicabilidad en productos vegetales todavía está en estudio. La termorresistencia de estos compuestos los convierte en un bioconservante ideal para la industria productora de vegetales enlatados o zumos tratados térmicamente (Komitopoulou, Boziaris, Davies, DelvesBroughton y Adams, 1999), siendo su capacidad de permanecer activas en ambientes ácidos (McAuliffe, Ross y Hill, 2001) lo que las hace interesantes como bioconservante en el sector de frutas y hortalizas mínimamente procesadas.

Existen distintos métodos que pueden emplearse para que las bacteriocinas Ileguen a los alimentos y actúen como bioconservantes. Uno de ellos es la adición del cultivo microbiano productor de bacteriocina a la matriz alimentaria, produciéndose de este modo las bacteriocinas in situ (Gragg y Brashears, 2010; Siroli et al., 2016) mientras que, por otro lado, puede cultivarse el microorganismo productor de bacteriocina en condiciones controladas para conseguir un purificado que contenga parcial o exclusivamente este compuesto que se podrá aplicar directamente en el alimento. Debido a que se ha descrito que la producción de bacteriocinas puede ser dependiente de la temperatura (Bennik, van Overbeek, Smid y Gorris, 1999), la aplicación directa del compuesto en el alimento permite tener un mayor control sobre la concentración de bacteriocina presente en el alimento y una distribución más homogénea del compuesto en el producto.

La nisina, bacteriocina producida por Lactococcus lactis subsp. lactis, es una de las bacteriocinas más estudiadas como bioconservante en frutas y hortalizas. Esta bacteriocina está aprobada como aditivo alimentario (E-314) y se comercializa a día de hoy bajo distintos nombres comerciales. En distintos estudios se ha mostrado su efectividad para controlar la población de Listeria monocytogenes en lechuga cortada (Oliveira et al., 2015; McManamon, Kaupper, Scollard y Schmalenberger, 2019; Allende et al., 2007). Del 
mismo modo, se ha determinado su capacidad para controlar la población de Salmonella spp. en el melón cortado (Ukuku y Fett, 2004), la microbiota natural del melón (Ukuku y Fett, 2002) y la población de Listeria monocytogenes y Escherichia coli 0157:H7 en el melón entero (Ukuku, Bari, Kawamoto y Isshiki, 2005). En vegetales como el calabacín o el brócoli se han observado reducciones de la población de Listeria monocytogenes superiores a 2,5 unidades logarítmicas con la aplicación de esta bacteriocina (Bari et al., 2005).

Cabe destacar que existen otras bacteriocinas como la coagulina (sobrenadante producido por Lactobacillus paraplantarum IPLA C23), pediocina (producida por Pediococcus acidilactici), bacteriocina RUC9 (producida por una cepa de Lactococcus lactis aislada de ensalada mínimamente procesada) o enterocina AS-48 (producida por Enterococcus faecalis A-48-32) que han mostrado ser efectivas para el control de Listeria monocytogenes en frutas y vegetales (Randazzo, Pitino, Scifò y Caggia, 2009; Cobo-Molinos et al., 2005; Allende et al. 2007). Sin embargo, estas bacteriocinas no se encuentran todavía autorizadas para su uso alimentario. Con el objetivo de incrementar la efectividad, tanto de nisina como de las otras bacteriocinas, en frutas y vegetales, algunos autores han evaluado su combinación con ácidos orgánicos o sustancias quelantes con capacidad antimicrobiana, viéndose incrementada su efectividad significativamente (Bari et al., 2005; Bennik et al., 1999; Cobo-Molinos et al., 2005; Ukuku et al., 2005).

Para incrementar la vida útil de frutas mínimamente procesadas algunos autores han aplicado películas comestibles como soporte de las bacteriocinas. Narsaiah et al. (2015) aplicaron un recubrimiento de alginato que incorporaba pediocina en papaya cortada, observando al finalizar los 21 días de conservación a $4^{\circ} \mathrm{C}$ una reducción cercana a 4,0 unidades logarítmicas de la población microbiana total. Barbosa, Araújo, Matos, Carneloss y Castro, (2013) aplicaron un recubrimiento de celulosa que incorporaba nisina en mango mínimamente procesado. El tratamiento redujo la población de Listeria. monocytogenes por debajo de los límites de detección después de cuatro días, mientras que en las muestras de control la población alcanzó $10^{6} \mathrm{ufc} \mathrm{g}^{-1}$. Resultados similares se observaron en la población de Staphylococcus aureus en mango a los seis días de conservación, con una reducción de 6 unidades logarítmicas. Sobre cubos de manzana inoculados con distintas cepas de Listeria monocytogenes, se evaluó la efectividad de distintos recubrimientos solos o combinados con enterocina AS-48 (López Aguayo, Grande Burgos, Pérez Pulido, Gálvez y Lucas López, 2016). Con el tra- tamiento combinado de carboximetil celulosa y enterocina $\left(40 \mu \mathrm{g} \mathrm{ml}^{-1}\right)$ se observó una reducción de 2,7 unidades logarítmicas a los siete días. Con la adición de lactato de sodio o EDTA a todas las combinaciones de recubrimientos y enterocina se consiguió una reducción de la población de Listeria monocytogenes por debajo de los límites de detección en todos los recubrimientos estudiados al cabo de un día con EDTA y a los siete días con el lactato de sodio.

\section{BACTERIÓFAGOS}

Los bacteriófagos o fagos son virus que infectan y se multiplican en bacterias específicas y son habitualmente inocuos para humanos, animales y plantas. Son los microorganismos más abundantes en el planeta y están ampliamente difundidos en aguas y alimentos de distintos orígenes (Brüssow y Kutter, 2005). Se clasifican en virulentos (líticos) que siguen generalmente un ciclo lítico y atemperados (lisogénicos) que entran e insertan su DNA en el interior del cromosoma bacteriano y son menos efectivos. El control biológico mediante fagos líticos está empezando a ser aceptado como una tecnología limpia para garantizar la seguridad alimentaria, ya que estos abordan muchas de las preocupaciones actuales de los consumidores. Por una parte, su especificidad hace que únicamente afecten a los microorganismos patógenos y no a la microbiota propia de los alimentos ni a la microbiota del tracto intestinal. Además, son respetuosos con el medioambiente, los fagos se han aislado de él. Los productos comerciales están formulados, en general, con soluciones acuosas, no contienen ni aditivos ni conservantes y su coste es relativamente bajo (Moye, Woolston y Sulakvelidze, 2018). No afectan a la calidad organoléptica del producto. Como inconvenientes, el hecho de que sean muy específicos es, por una parte, ventajoso, pero también puede ser una desventaja; para hacer frente a varios patógenos se necesitará utilizar varios fagos. Además, han de conservarse en refrigeración y su fecha de caducidad es más limitada que la de otros productos.

Existen numerosos estudios que han demostrado la efectividad de determinados bacteriófagos en frutas y hortalizas procesadas. El cóctel de bacteriófagos ECP-100 (actualmente EcoShield ${ }^{\mathrm{TM}}$ ) ha demostrado ser efectivo frente a Escherichia coli 0157:H7 en tomates, brócoli o espinacas (Abdulaze et al., 2008), lechuga y melón cortado (Sharma, Patel, Conway, Ferguson y Sulakvelidze, 2009). El uso de atmósferas modificadas mejoró la efectividad de este fago (Boyacioglu, Sharma, Sulakvelidze y Goktepe, 2013) en lechuga y 
espinacas. De forma similar, el bacteriófago LMP-102 (actualmente ListShield ${ }^{\mathrm{TM}}$ ), efectivo frente a Listeria monocytogenes, se ha ensayado en melón y manzana cortada conservados a $10{ }^{\circ} \mathrm{C}$, mostrando reducciones entre 2 y 0,4 unidades logarítmicas (Leverentz et al., 2003), respectivamente. La efectividad de este producto también se ha probado frente a biofilms sobre lechuga, y superficies de acero inoxidable y caucho, reduciéndolo significativamente en todos los casos. Cabe destacar este efecto en biofilms, ya que estos son difícilmente eliminables mediante otros desinfectantes. Nuestro grupo de investigación evaluó la efectividad del fago Listex P100 frente a manzana, pera y melón cortados y en sus zumos (Oliveira et al., 2014). Los resultados demostraron que, tras ocho días de conservación a $10^{\circ} \mathrm{C}$, el fago no fue efectivo en manzana cortada ni en su zumo, seguramente debido al bajo $\mathrm{pH}$ de la manzana que hizo decrecer rápidamente el número de fagos presentes. En pera y melón cortado se obtuvieron reducciones de 1,5 y 1,0 unidades logarítmicas, respectivamente y entre 8,0 y 2,1 unidades en sus zumos. Estas diferencias pueden atribuirse a que, en matrices sólidas, el fago quede inmovilizado y no pueda entrar en contacto con el patógeno debido a su limitada difusión. En lo que se refiere a Salmonella spp. (Leverentz et al., 2001), el fago SCPLX-1 fue efectivo en melón, pero no en manzana. En brotes de soja verde y de semillas de alfalfa, un cóctel de fagos aislados de efluentes de granjas de cerdo y ganado redujeron 3 unidades logarítmicas la población de Salmonella spp., incrementándose esta reducción cuan- do el fago se combinó con bacterias antagonistas (Ye, Kostrzynska, Dunfield y Warriner, 2010). En este sentido, Leverentz et al. (2003) demostraron también que la combinación del fago LMP-102 con nisina mejoró su efectividad frente a Listeria monocytogenes en melón. La combinación de los fagos con un lavado también puede incrementar su efectividad. Así, Magnone, Marek, Sulakvelidze y Senecal (2013) demostraron que el lavado con ácido levulínico y un cóctel de bacteriófagos (EcoShield ${ }^{\mathrm{TM}}$, SalmoFresh ${ }^{\mathrm{TM}}$ y ShigaActive ${ }^{\mathrm{TM}}$ ), incrementaron la efectividad del tratamiento exclusivo con los fagos en fresa, brócoli y melón cantaloupe. En lechuga cortada Oliveira et al. (2015) compararon la efectividad de distintos bioconservantes frente a Salmonella y Listeria monocytogenes. Ni el fago SalmoneIlex ni los cultivos bioconservantes ensayados redujeron la población de Salmonella, que no creció durante seis días a $10^{\circ} \mathrm{C}$. Los fagos también pueden incorporarse en recubrimientos alimentarios (Vonasek, Choi, Sanchez y Nitin 2018) con el fin de albergar más fagos y mejorar su estabilidad durante la conservación.

Actualmente, algunos bacteriófagos ya están aprobados para su uso en frutas y hortalizas (véase la tabla 1). En los Estados Unidos, Canadá e Israel prácticamente están todos aprobados, mientras que en la Unión Europea están permitidos como coadyuvantes tecnológicos. El panel de riesgos biológicos de la EFSA (BIOHAZ) emitió una opinión científica sobre el uso de Listex P100 en alimentos listos para el consumo (EFSA, Panel on Biological Hazards (BIOHAZ), 2016).

Tabla 1. Productos a base de bacteriófagos aprobados para su aplicación en frutas y hortalizas

\begin{tabular}{|c|c|c|c|c|}
\hline Empresa & Producto & Organismo diana & Países autorizados & Certificaciones \\
\hline \multirow{4}{*}{$\begin{array}{l}\text { Intralytix, Inc. } \\
\text { (Baltimore, MD, } \\
\text { USA) }\end{array}$} & $\begin{array}{l}\text { EcoShield }{ }^{\mathrm{TM}} \\
\text { (anteriormente ECP-100) }\end{array}$ & E. coli 0157:H7 & EEUU, Israel, Canadá & Kosher; Halal \\
\hline & $\begin{array}{l}\text { ListShield }^{\mathrm{TM}} \\
\text { (anteriormente LMP-102) }\end{array}$ & L. monocytogenes & EEUU, Israel, Canadá & Kosher, Halal, OMRI \\
\hline & SalmoFresh ${ }^{\mathrm{TM}}$ & Salmonella spp. & EEUU, Israel, Canadá & Kosher, Halal, OMRI \\
\hline & ShigaShield $^{\mathrm{TM}}$ (ShigActive $^{\mathrm{TM}}$ ) & Shigella spp. & EEUU & \\
\hline \multirow{3}{*}{$\begin{array}{l}\text { PhageGuard } \\
\text { (Micreos } \\
\text { Food Safety, } \\
\text { Wageningen, } \\
\text { Netherlands) }\end{array}$} & $\begin{array}{l}\text { PhageGuard Listex }{ }^{\mathrm{TM}} \\
\text { (anteriormente Listex P100) }\end{array}$ & L. monocytogenes & $\begin{array}{l}\text { EEUU, EU, Canadá, } \\
\text { Australia, Nueva Zelanda, } \\
\text { Suiza y Israel }\end{array}$ & $\begin{array}{l}\text { Kosher, Halal, } \\
\text { OMRI, SKAL }\end{array}$ \\
\hline & $\begin{array}{l}\text { PhageGuard } \mathrm{S}^{\mathrm{TM}} \\
\text { (anteriormente Salmonellex) }\end{array}$ & Salmonella spp. & EEUU, Suiza, Israel, Canadá & $\begin{array}{l}\text { Kosher, Halal, } \\
\text { OMRI, SKAL }\end{array}$ \\
\hline & PhageGuard E & E. coli 0157:H7 & $\begin{array}{l}\text { EEUU (autorizado para } \\
\text { otros alimentos, pendiente } \\
\text { aprobación en vegetales) }\end{array}$ & \\
\hline
\end{tabular}

Fuente: adaptado de Moye et al., 2018. 


\section{CONCLUSIONES}

Con los métodos utilizados actualmente en la industria de IV gama es difícil obtener frutas y hortalizas mínimamente procesadas totalmente seguras, lo que queda demostrado por el aumento de las toxiinfecciones asociadas al consumo de este tipo de productos. Además, los consumidores demandan productos libres de conservantes químicos. Por ello, la industria necesita implementar nuevas estrategias introduciendo o combinando técnicas sostenibles con las prácticas habituales llevadas a cabo, como la desinfección con hipoclorito sódico, el envasado en atmósferas modificadas y la conservación a temperaturas de refrigeración. La bioconservación, ya sea mediante el uso de cultivos microbianos o sus metabolitos, encaja perfectamente en esta necesidad de la industria.

El uso de cultivos protectores puede reducir el riesgo microbiológico asociado al consumo de frutas y hortalizas mínimamente procesadas y podría, además, alargar su vida útil. Sin embargo, la aplicación de estos cultivos no tiene un efecto inmediato sobre la población de patógenos de transmisión alimentaria, sino que ejerce un efecto protector frente al crecimiento de los mismos durante la conservación del producto. Por lo tanto, su uso debería realizarse en combinación con las actuales técnicas de producción utilizadas habitualmente en productos de IV gama. Además, diversos estudios han demostrado que la presencia de estos cultivos protectores disminuye la capacidad de los patógenos para adherirse e invadir el epitelio intestinal. Con todo, existen una serie de obstáculos para la aplicación práctica de los agentes de biocontrol, entre los que podemos destacar la aceptación por parte del consumidor y los aspectos legislativos. La presencia de microorganismos vivos en los alimentos no es una idea nueva, ya que desde tiempos muy antiguos se han utilizado para conservar alimentos mediante las fermentaciones, por lo que podría ser viable la aceptación de su uso por parte de los consumidores. No obstante, su aceptación legislativa y que obtenga el estatus QPS (Qualified Presumption of Safety), requerido para su autorización en el mercado europeo, pueden ser más complicados, necesitándose estudios sobre su toxicidad y patogenicidad.

Como se ha descrito en multitud de estudios, la aplicación de bacteriocinas en frutas y vegetales no ocasiona ningún defecto visible, siendo capaz de controlar tanto a los microorganismos alterantes como a los patógenos, por lo que se reafirma nuevamente su aptitud como bioconservante en este tipo de alimentos.

En cuanto a los fagos, su efectividad depende de la matriz, del momento y modo de aplicación y de su estabilidad durante la conservación, y su efecto no es inmediato. Así pues, aunque existen aún algunos retos por superar, el control biológico mediante fagos puede ser una tecnología efectiva para la eliminación o reducción de bacterias patógenas que no afecta a otros microorganismos. Finalmente, la aplicación de los fagos no ha de ser un sustituto del lavado, sino que ha de considerarse una tecnología de barrera adicional para garantizar la seguridad de frutas y hortalizas procesadas durante su comercialización.

\section{AGRADECIMIENTOS}

Los autores agradecen a MINECO por su soporte económico (Proyectos AGL-2004-06027, AGL-200908506 y AGL-2012-38671), a FECYT por la financiación del proyecto FECYT CC/2013105 y al Programa CERCA de la Generalitat de Catalunya.

\section{BIBLIOGRAFÍA}

Abadias, M., Altisent, R., Usall, J., Torres, R., Oliveira, M. y Viñas, I. (2014). Biopreservation of fresh-cut melon using the strain Pseudomonas graminis CPA-7. Postharvest Biology and Technology, 96, pp. 69-77. https://doi.org/10.1016/j. postharvbio.2014.05.010

Abadias, M., Usall, J., Alegre, I., Torres, R. y Viñas, I. (2009). Fate of Escherichia coli in apple and reduction of its growth using the postharvest biocontrol agent Candida sake CPA-1. Journal of the Science of Food and Agriculture, 89 (9), pp. 1526 1533. https://doi.org/10.1002/jsfa.3619
Abuladze, T., Li, M., Menetrez, M. Y., Dean, T., Senecal, A. y Sulakvelidze, A. (2008). Bacteriophages reduce experimental contamination of hard surfaces, tomato, spinach, broccoli, and ground beef by Escherichia coli 0157:H7. Applied and Environmental Microbiology, 74 (20), pp. 6230-6238. https://doi. org/10.1128/AEM.01465-08

Alegre, I., Viñas, I., Usall, J., Anguera, M. y Abadias, M. (2011). Microbiological and physicochemical quality of fresh-cut apple enriched with the probiotic strain Lactobacillus rhamnosus GG. Food Mi- crobiology, 28 (1), pp. 59-66. https:// doi.org/10.1016/j.fm.2010.08.006

Alegre, I., Viñas, I., Usall, J., Anguera, M., Altisent, R. y Abadias, M. (2013). Antagonistic effect of Pseudomonas graminis CPA-7 against foodborne pathogens in freshcut apples under simulated commercial conditions. Food Microbiology, 33 (2), pp. 139-148. https://doi.org/10.1016/j. fm.2012.09.007

Alegre, I., Viñas, I., Usall, J., Anguera, M., Figge, M. J., Abadias, M. (2012). An Enterobacteriaceae species isolated from 
apples controls foodborne pathogens on fresh-cut apples and peaches. Postharvest Biology and Technology, 74, pp. 118-124. https://doi.org/10.1016/j. postharvbio.2012.07.004

Alegre, I., Viñas, I., Usall, J., Teixidó, N., Figge, M. J. y Abadias, M. (2013). Control of foodborne pathogens on fresh-cut fruit by a novel strain of Pseudomonas graminis. Food Microbiology, 34, pp. 390-399. https://doi.org/10.1016/j.fm.2013.01.013

Allende, A., Martínez, B., Selma, V., Gil, M. I., Suárez, J. E. y Rodríguez, A. (2007). Growth and bacteriocin production by lactic acid bacteria in vegetable broth and their effectiveness at reducing Listeria monocytogenes in vitro and in fresh-cut lettuce. Food Microbiology, 24 (7-8), pp. 759-766. https://doi. org/10.1016/j.fm.2007.03.002

Artés, F., Gómez, P., Aguayo, E., Escalona, V. y Artés-Hernández, F. (2009). Sustainable sanitation techniques for keeping quality and safety of fresh-cut plant commodities. Postharvest Biology and Technology, 51 (3), pp. 287-296. https://doi.org/10.1016/j.postharvbio.2008.10.003

Barbosa, A. A. T., Araújo, H. G. S. de, Matos, P. N., Carneloss, M. A. G. y Castro, A. A. de (2013). Effects of nisin-incorporated films on the microbiological and physicochemical quality of minimally processed mangoes. International Journal of Food Microbiology, 164 (2-3), pp. 135-140. https://doi.org/10.1016/j.ijfoodmicro.2013.04.004

Bari, M. L., Ukuku, D. O., Kawasaki, T., Inatsu, Y., Isshiki, K. y Kawamoto, S. (2005). Combined efficacy of nisin and pediocin with sodium lactate, citric acid, phytic acid, and potassium sorbate and EDTA in reducing the Listeria monocytogenes population of inoculated fresh-cut produce. Journal of Food Protection, 68 (7), pp. 1381-1387. https://doi. org/10.4315/0362-028x-68.7.1381

Bennik, M. H. J., van Overbeek, W., Smid, E. J. y Gorris, L. G. M. (1999). Biopreservation in modified atmosphere stored mungbean sprouts: The use of vegetable-associated bacteriocinogenic lactic acid bacteria to control the growth of Listeria monocytogenes. Letters in Applied Microbiology, 28 (3), pp. 226232. https://doi.org/10.1046/j.13652672.1999.00497.x

Boyacioglu, O., Sharma, M., Sulakvelidze, A. y Goktepe, I. (2013). Biocontrol of Esch- erichia coli $0157: \mathrm{H} 7$ on fresh-cut leafy greens. Bacteriophage, 3 (1), e24620. https://doi.org/10.4161/bact.24620

Brüssow, H. y Kutter, E. (2005). Phage ecology. En: Kutter, E. y Sulakvelidze, A. (eds.). Bacteriophages: biology and applications. Florida: Boca Raton CRC Press, pp. 129-163.

Chen, H. y Hoover, D. G. (2003). Bacteriocins and their food applications. Comprehensive Reviews in Food Science and Food Safety, 2 (3), pp. 82-100. https:// doi.org/10.1111/j.1541-4337.2003. tb00016.x

Cobo-Molinos, A., Abriouel, H., Ben Omar, N., Valdivia, E., López, R. L., Maqueda, M., Martínez Cañamero, M. y Gálvez, A. (2005). Effect of immersion solutions containing enterocin AS-48 on Listeria monocytogenes in vegetable foods. Applied and Environmental Micorbiology, 71 (12), pp. 7781-7787. https://doi.org/10.1128/ AEM.71.12.7781-7787.2005

Collazo, C., Abadias, M., Aguiló-Aguayo, I., Alegre, I., Chenoll, E. y Viñas, I. (2017). Studies on the biocontrol mechanisms of Pseudomonas graminis strain CPA7 against food-borne pathogens in vitro and on fresh-cut melon. LWT Food Science and Technology, 85, pp. 301-308. https://doi.org/10.1016/j. Iwt.2017.02.029

Collazo, C., Abadias, M., Colás-Medà, P., Iglesias, M. B., Granado-Serrano, A. B., Serrano, J. y Viñas, I. (2017). Effect of Pseudomonas graminis strain CPA-7 on the ability of Listeria monocytogenes and Salmonella enterica subsp. enterica to colonize Caco-2 cells after pre-incubation on fresh-cut pear. International Journal of Food Microbiology, 262, pp. 55-62. https://doi.org/10.1016/j.ijfoodmicro.2017.09.003

Collazo, C., Giné-Bordonaba, J., AguilóAguayo, I., Povedano, I., Bademunt, A. y Viñas, I. (2018). Pseudomonas graminis strain CPA-7 differentially modulates the oxidative response in fresh-cut 'Golden delicious' apple depending on the storage conditions. Postharvest Biology and Technology, 138, pp. 4655. https://doi.org/10.1016/j.postharvbio.2017.12.013

Cotter, P. D., Hill, C. y Ross, P. (2005). Bacteriocins: developing innate immunity for food. Nature Reviews. Microbiology, 3 (10), pp. 777-788. https://doi. org/10.1038/nrmicro1273
European Food Safety Authority and European Centre for Disease Prevention and Control (EFSA and ECDC) (2018). The European Union summary report on trends and sources of zoonoses, zoonotic agents and food-borne outbreaks in 2017. EFSA Journal, 16 (12), e05500. https://doi. org/10.2903/j.efsa.2018.5500

EFSA Panel on Biological Hazards (BIOHAZ) (2016). Evaluation of the safety and efficacy of Listex ${ }^{\mathrm{TM}}$ P100 for reduction of pathogens on different ready-to-eat (RTE) food products. EFSA Journal, 14 (8), e04565. https://doi.org/10.2903/j. efsa.2016.4565

Faleiro, M. L. (2010). The mode of antibacterial action of essential oils. Science against microbial pathogens: communicating current research and technological advances, 2, pp. 1143-1156.

Fliss, I., Hammami, R. y Le Lay, C. (2011). Biological control of human digestive microbiota using antimicrobial cultures and bacteriocins. En Lacroix, C (ed.). Protective cultures, antimicrobial metabolites and bacteriophages for food and beverage biopreservation. Cambridge, U. K.: Woodhead Publishing, pp. 240-263.

Francis, G. A. y O’Beirne, D. (1997). Effects of gas atmosphere, antimicrobial dip and temperature on the fate of Listeria innocua and Listeria monocytogenes on minimally processed lettuce. International Journal of Food Science and Technology, 32 (2), pp. 141-151. https://doi. org/10.1046/j.1365-2621.1997.00390.x

Gil, M. I., Selma, M. V., López-Gálvez, F. y Allende, A. (2009). Fresh-cut product sanitation and wash water disinfection: Problems and solutions. International Journal of Food Microbiology, 134 (1-2), pp. 37-45. https://doi.org/10.1016/j.ijfoodmicro.2009.05.021

Gragg, S. E. y Brashears, M. M. (2010). Reduction of Escherichia coli 0157:H7 in fresh spinach, using lactic acid bacteria and chlorine as a multihurdle intervention. Journal of Food Protection 73 (2), pp. 358-361. https://doi. org/10.4315/0362-028x-73.2.358

Holzapfel, W. H., Geisen, R. y Schillinger, U. (1995). Biological preservation of foods with reference to protective cultures, bacteriocins and food-grade enzymes. International Journal of Food Microbiology, 24 (3), pp. 343-362. https://doi. org/10.1016/0168-1605(94)00036-6 
Iglesias, M. B., Abadias, M., Anguera, M., Sabata, J. y Viñas, I. (2017). Antagonistic effect of probiotic bacteria against foodborne pathogens on fresh-cut pear. LWT-Food Science and Technology, 81, pp. 243-249. https://doi.org/10.1016/j. Iwt.2017.03.057

Iglesias, M. B., Abadias, M., Anguera, M. y Viñas, I. (2018). Efficacy of Pseudomonas graminis CPA-7 against Salmonella spp. and Listeria monocytogenes on fresh-cut pear and setting up of the conditions for its commercial application. Food Microbiology, 70, pp. 103-112. https://doi.org/10.1016/j. fm.2017.09.010

Iglesias, M. B., Echeverría, G., Viñas, I., López, M. L. y Abadias, M. (2018). Biopreservation of fresh-cut pear using Lactobacillus rhamnosus GG and effect on quality and volatile compounds. LWT - Food Science and Technology, 87, pp. 581-588. https://doi.org/10.1016/j. Iwt.2017.09.025

Iglesias, M. B., López, M. L., Echeverría, G., Viñas, I., Zudaire, L. y Abadias, M. (2018). Evaluation of biocontrol capacity of Pseudomonas graminis CPA-7 against foodborne pathogens on freshcut pear and its effect on fruit volatile compounds. Food Microbiology, 76, pp. 226-236. https://doi.org/10.1016/j. fm.2018.04.007

Iglesias, M. B., Viñas, I., Colás-Medà, P., Collazo, C., Serrano, J. C. E. y Abadias, M. (2017). Adhesion and invasion of Listeria monocytogenes and interaction with Lactobacillus rhamnosus GG after habituation on fresh-cut pear. Journal of Functional Foods, 34, pp. 453-460. https://doi.org/10.1016/j. jff.2017.05.011

Komitopoulou, E., Boziaris, I. S., Davies, E. A., Delves-Broughton, J. y Adams, M. R. (1999). Alicyclobacillus acidoterrestris in fruit juices and its control by nisin. International Journal of Food Science and Technology, 34 (1), pp. 81-85. https://doi.org/10.1046/j.13652621.1999.00243.x

Leverentz, B., Conway, W. S., Alavidze, Z., Janisiewicz, W. J., Fuchs, Y., Camp, M. J., Chighladze, E. y Sulakvelidze, A. (2001). Examination of bacteriophage as a biocontrol method for Salmonella on fresh-cut fruit: A model study. Journal of Food Protection, 64 (8), pp. 11161121. https://doi.org/10.4315/0362028x-64.8.1116
Leverentz, B., Conway, W. S., Camp, M. J., Janisiewicz, W. J., Abuladze, T., Yang, M., Saftner, R. y Sulakvelidze, A. (2003). Biocontrol of Listeria monocytogenes on fresh-cut produce by treatment with lytic bacteriophages and a bacteriocin. Applied and Environmental Microbiology, 69 (8), pp. 4519-4526. https:// doi.org/10.1128/AEM.69.8.45194526.2003

Leverentz, B., Conway, W. S., Janisiewicz, W., Abadias, M., Kurtzman, C. P. y Camp, M. J. (2006). Biocontrol of the food-borne pathogens Listeria monocytogenes and Salmonella enterica serovar Poona on fresh-cut apples with naturally occurring bacterial and yeast antagonists. Applied and Environmental Microbiology, 72 (2), pp. 11351140. https://dx.doi.org/10.1128\%2FA EM.72.2.1135-1140.2006

Liao, C. y Fett, W. F. (2001). Analysis of native microflora and selection of strains antagonistic to human pathogens on fresh produce. Journal of Food Protection, 64 (8), pp. 1110-1115. https://doi. org/10.4315/0362-028x-64.8.1110

López Aguayo, M. D. C., Grande Burgos, M. J., Pérez Pulido, R., Gálvez, A. y Lucas López, R. (2016). Effect of different activated coatings containing enterocin AS-48 against Listeria monocytogenes on apple cubes. Innovative Food Science and Emerging Technologies, 35, pp. 177-183. https://doi.org/10.1016/j. ifset.2016.05.006

Magnone, J. P., Marek, P. J., Sulakvelidze, A. y Senecal, A. G. (2013). Additive approach for inactivation of Escherichia coli 0157:H7, Salmonella, and Shigella spp. on contaminated fresh fruits and vegetables using bacteriophage cocktail and produce wash. Journal of Food Protection, 76 (8), pp. 1336-1341. https://doi. org/10.4315/0362-028X.JFP-12-517

McAuliffe, O., Ross, R. y Hill, C. (2001). Lantibiotics: structure, biosynthesis and mode of action. FEMS Microbiology Reviews, 25 (3), pp. 285-308. https:// doi.org/10.1111/j.1574-6976.2001. tb00579.x

McManamon, O., Kaupper, T., Scollard, J. y Schmalenberger, A. (2019). Nisin application delays growth of Listeria monocytogenes on fresh-cut iceberg lettuce in modified atmosphere packaging, while the bacterial community structure changes within one week of storage. Postharvest Biology and Tech- nology, 147, pp. 185-195. https://doi. org/10.1016/j.postharvbio.2018.10.002

Mondragón Preciado, G., Escalante Minakata, P., Osuna Castro, J. A., Ibarra Junquera, V. I., Morlett Chávez, J. A., Aguilar González, C. N. y Rodriguez Herrera, R. (2013). Bacteriocinas: características y aplicación en alimentos. Investigación y Ciencia de La Universidad Autónoma de Aguascalientes, 21 (59), pp. 63-69.

Moye, Z. D., Woolston, J. y Sulakvelidze, A. (2018). Bacteriophage applications for food production and processing. Viruses, 10 (4), pp. 205. https://dx.doi. org/10.3390\%2Fv10040205

Narsaiah, K., Wilson, R. A., Gokul, K., Mandge, H. M., Jha, S. N., Bhadwal, S., Anurag, R. K., Malik, R, K. [...] y Vij, S. (2015). Effect of bacteriocin-incorporated alginate coating on shelf-life of minimally processed papaya (Carica papaya L.). Postharvest Biology and Technology, 100, pp. 212-218. https://doi. org/10.1016/j.postharvbio.2014.10.003

Oliveira, M., Abadias, M., Colás-Medà, P., Usall, J. y Viñas, I. (2015). Biopreservative methods to control the growth of foodborne pathogens on fresh-cut lettuce. International Journal of Food Microbiology, 214, pp. 4-11. https://doi. org/10.1016/j.ijfoodmicro.2015.07.015

Oliveira, M., Viñas, I., Colàs, P., Anguera, M., Usall, J. y Abadias, M. (2014). Effectiveness of a bacteriophage in reducing Listeria monocytogenes on fresh-cut fruits and fruit juices. Food Microbiology, 38, pp. 137-142. https://doi.org/10.1016/j. fm.2013.08.018

Ongeng, D., Ryckeboer, J., Vermeulen, A. y Devlieghere, F. (2007). The effect of micro-architectural structure of cabbage substratum and or background bacterial flora on the growth of Listeria monocytogenes. International Journal of Food Microbiology, 119 (3), pp. 291299. https://doi.org/10.1016/j.ijfoodmicro.2007.08.022

Ramos, B., Miller, F. A., Brandao, T. R. S., Teixeria, P. y Silva, C. L. M. (2013). Fresh fruits and vegetables: an overview on applied methodologies to improve its quality and safety. Innovative Food Science and Emerging Technologies, 20, pp. 1-15. https://doi.org/10.1016/j.ifset.2013.07.002

Randazzo, C. L., Pitino, I., Scifò, G. O. y Caggia, C. (2009). Biopreservation of minimally processed iceberg lettuces using 
a bacteriocin produced by Lactococcus lactis wild strain. Food Control, 20 (8), pp. 756-763. https://doi.org/10.1016/j. foodcont.2008.09.020

Russo, P., de Chiara, M. L. V., Vernile, A., Amodio, M. L., Arena, M. P., Capozzi, V., Massa, S. y Spano, G. (2014). Fresh-cut pineapple as a new carrier of probiotic lactic acid bacteria. BioMed Research International, 2014, 309183. http:// dx.doi.org/10.1155/2014/309183

Russo, P., Peña, N., de Chiara, M. L. V., Amodio, M. L., Colelli, G. y Spano, G. (2015). Probiotic lactic acid bacteria for the production of multifunctional fresh-cut cantaloupe. Food Research International, 77, pp. 762-772. https:// doi.org/10.1016/j.foodres.2015.08.033

Sánchez, G., Elizaquível, P. y Aznar, R. (2012). A single method for recovery and concentration of enteric viruses and bacteria from fresh-cut vegetables. International Journal of Food Microbiology, 152 (1-2), pp. 9-13. https://doi. org/10.1016/j.ijfoodmicro.2011.10.002

Scolari, G. y Vescovo, M. (2004). Microbial antagonism of Lactobacillus casei added to fresh vegetables. Italian Journal of Food Science, 16 (4), pp. 465-475.

Sharma, M., Patel, J. R., Conway, W. S., Ferguson, S. y Sulakvelidze, A. (2009). Effectiveness of bacteriophages in reducing Escherichia coli 0157:H7 on fresh-cut cantaloupes and lettuce. Journal of Food Protection, 72 (7), pp. 1481-1485. https:// doi.org/10.4315/0362-028x-72.7.1481

Siroli, L., Patrignani, F., Serrazanetti, D. I., Tabanelli, G., Montanari, C., Gardini, F. y Lanciotti, R. (2015). Lactic acid bacteria and natural antimicrobials to improve the safety and shelf-life of minimally processed sliced apples and lamb's lettuce. Food Microbiology, 47, pp. 74-84. https://doi.org/10.1016/j. fm.2014.11.008

Siroli, L., Patrignani, F., Serrazanetti, D. I., Vannini, L., Salvetti, E., Torriani, S., Gardini, F. y Lanciotti, R. (2016). Use of a nisin-producing Lactococcus lactis strain, combined with natural antimicrobials, to improve the safety and shelf-life of minimally processed sliced apples. Food Microbiology, 54, pp. 11-19. https:// doi.org/10.1016/j.fm.2015.11.004

Torriani, S., Scolari, G., Dellaglio, F. y Vescovo, M. (1999). Biocontrol of leuconostocs in ready-to-use shredded carrots. Annali di Microbiologia ed Enzimologia, 49, pp. 23-31.

Trias, R., Bañeras, L., Badosa, E. y Montesinos, E. (2008). Bioprotection of Golden Delicious apples and Iceberg lettuce against foodborne bacterial pathogens by lactic acid bacteria. International Journal of Food Microbiology, 123 (1-2), pp. 50-60. https://doi.org/10.1016/j. ijfoodmicro.2007.11.065

Ukuku, D. O., Bari, M. L., Kawamoto, S. y Isshiki, K. (2005). Use of hydrogen peroxide in combination with nisin, sodium lactate and citric acid for reducing transfer of bacterial pathogens from whole melon surfaces to fresh-cut pieces. International Journal of Food Microbiology, 104 (2), pp. 225-233. https://doi. org/10.1016/j.ijfoodmicro.2005.01.016

Ukuku, D. O. y Fett, W. F. (2002). Effectiveness of chlorine and nisin-EDTA treatments of whole melons and fresh-cut pieces for reducing native microflora and extending shelf-life. Journal Food Safety, 22 (4), pp. 231-253. https:// doi.org/10.1111/j.1745-4565.2002. tb00344.x

Ukuku, D. O. y Fett, W. F. (2004). Effect of nisin in combination with EDTA, sodium lactate, porassium sorbate for reducing Salmonella on whole fresh-cut cantaloupe. Journal of Food Protection, 67 (10), pp. 2143-2150. https://doi. org/10.4315/0362-028x-67.10.2143

Vescovo, M., Torriani, S., Orsi, C., Macchiarolo, F. y Scolari, G. (1996). Application of antimicrobial-producing lactic acid bacteria to control pathogens in ready-touse vegetables. Journal of Applied Bacteriology, 81 (2), pp. 113-119. https:// doi.org/10.1111/j.1365-2672.1996. tb04487.x
Viñas, I., Abadias, M., Usall, J., Teixido, N. y Torres, R. (2017). UE Patente No EP2886665. Lleida, España. European Patent Office.

Viñas, I., Abadias, M., Usall, J., Teixidó, N. y Torres, R. (2014). EE. UU. Patente № 8735136. United States Patent and Trademark Office.

Vonasek, E. L., Choi, A. H., Sanchez, J. Jr. y Nitin, N. (2018). Incorporating phage therapy into WPI dip coatings for applications on fresh whole and cut fruit and vegetable surfaces. Journal of Food Science, 83 (7), pp. 1871-1879. https:// doi.org/10.1111/1750-3841.14188

Wang, X., Ouyang, Y., Liu, J., Zhu, M., Zhao, G., Bao, W. y Hu, F. B. (2014). Fruit and vegetable consumption and mortality from all causes, cardiovascular disease, and cancer: systematic review and dose-response meta-analysis of prospective cohort studies. BMJ: British Medical Journal, 349, g4490. https:// doi.org/10.1136/bmj.g4490

Weiss, A., Hertel, C., Grothe, S., Ha, D. y Hammes, W. P. (2007). Characterization of the cultivable microbiota of sprouts and their potential for application as protective cultures. Systematic and Applied Microbiology, 30 (6), pp. 483-493. https://doi.org/10.1016/j.syapm.2007.03.006

Ye, J., Kostrzynska, M., Dunfield, K. y Warriner, K. (2010). Control of Salmonella on sprouting mung bean and alfalfa seeds by using a biocontrol preparation based on antagonistic bacteria and lytic bacteriophages. Journal of Food Protection, 73 (1), pp. 9-17. https://doi. org/10.4315/0362-028x-73.1.9

\section{Recursos en línea}

La IV gama de frutas y hortalizas espera crecer un $10 \%$ hasta 2020. Valencia Fruits. [En línea]. Disponible en: http:// valenciafruits.com/la-iv-gama-defrutas-y-hortalizas-espera-crecer-un10-hasta-2020/ 